\title{
Effects of housing space and litter size on play behavior in rats
}

\author{
HEATHER J. KLINGER and ERNEST D. KEMBLE \\ University of Minnesota, Morris, Minnesota
}

\begin{abstract}
The effect of housing space and litter size on the play behavior of young rats was investigated in two experiments. Male play increased steadily with increasing space, whereas female play decreased in the largest environment. These effects indicate a sex-dependent suppression of play by limited housing space. When litter size was reduced at the time of weaning, the play of the split litters differed from that of litters comparable in size or housing space. This suggests that litter size may also be an important determinant of play in young rats.
\end{abstract}

Play behavior is strongly influenced by age (Meany \& Stewart, 1981), social isolation (Panksepp \& Beatty, 1980), sex of play partner (Thor \& Holloway, 1983), and early suckling experience (Koepke \& Pribram, 1971). A recent study also suggested that play was strongly influenced by litter size in grasshopper mice (Davies \& Kemble, 1983), with litters of three mice playing more than twice as much as litters of four to six individuals. Although it is tempting to attribute this effect to the differential experiences of mice in small or large litters, all litters in the previous study were reared in identical, rather small aquaria. Thus, it is also possible that decreased play resulted from the more restricted space per individual in larger litters. In either case, it seemed of interest to explore this phenomenon further. The present experiments assessed the effects of litter size and housing space on the play behavior of albino rats.

\section{EXPERIMENT 1}

The first experiment sought to replicate and extend the litter-size effect reported for $O$. leucogaster (Davies \& Kemble, 1983). Small $(\mathrm{N}=4)$ and large $(\mathrm{N}=8)$ litters were reared in identical aquaria, and their play was observed following weaning. To determine whether lower levels of play in large litters would reflect the suppressive effects of restricted space, one large litter was divided into two small litters at weaning, and each group was separately housed prior to observation.

\section{Method}

Subjects. The subjects were six litters of rats derived from females supplied by the Holtzman Company. The litters were reduced in size to two litters of eight (male $=4$, female $=4$ ) and four litters of four (male $=2$, female $=2$ ) when the rats were 4-10 days of age. Litters were born and reared in $50 \times 27 \times 31 \mathrm{~cm}$ aquaria containing commercial clay litter and had ad-lib access to Purina Lab Chow and water throughout the experiment. The subjects were weighed at 30 days of

Please send reprint requests to Ernest Kemble, Division of Social Sciences, University of Minnesota-Morris, Morris, MN 56267. age, tails marked for identification, and the mothers removed from the aquaria. One litter of eight (split litter) was divided into two groups of four (male $=2$, female $=2$ ), and each group was separately housed in $50 \times 27 \times 31 \mathrm{~cm}$ aquaria at the time of weaning. The subjects were maintained on a 12 -h light $(0800 \mathrm{~h}-2000 \mathrm{~h}) /$ dark cycle throughout the experiments. Two $25-\mathrm{W}$ red incandescent bulbs provided dim illumination during the dark cycle. Duration of observation intervals was controlled by $28-\mathrm{V}$ dc programming equipment, and duration of play was recorded on a 0.01 -sec electronic timer.

Procedure. Five days after the rats were weaned, play behavior in the intact litters was measured on 4 consecutive days. The observer entered the room at the onset of the dark cycle and allowed 2-3 min for adaptation. Each rat was observed for five 60 -sec intervals daily. Each observation period was separated by observations of all other rats, and the order of observation was balanced across days. During each interval, the frequencies of wrestling, boxing, pouncing, chasing, pinning, and aggressive grooming were recorded (see Davies \& Kemble, 1983 , and Meany \& Stewart, 1981, for descriptions of the behaviors). Duration of play and instances of social grooming were also recorded.

\section{Results}

Initial analyses compared the frequency and duration of all scored play behaviors combined and summed across the 4 observation days. Frequency (upper panel) and duration (lower panel) of play are summarized in Figure 1. Analysis of variance revealed a highly significant effect of litter size on play frequency $[F(2,26)=15.16$, $\mathrm{p}<.001$ ]. Individual comparisons showed that both small $(\mathrm{p}<.01)$ and split litters $(\mathrm{p}<.01)$ played more frequently than the large litter. Furthermore, the split litter played more frequently than the small litters $(\mathrm{p}<.05)$. There was no overall sex difference in play frequency and no sex $\times$ litter size interaction (ps $<.10)$. Litter size also strongly affected the frequency of social grooming in large (mean $=1.75)$, small (mean $=6.94)$, and split litters (mean $=3.75$ ) during observations $[\mathrm{F}(2,26)=7.42$, $p<.005]$. Small litters engaged in more social grooming than either large $(\mathrm{p}<.05)$ or split litters $(\mathrm{p}<.05)$, but there was no reliable difference between large and split litters $(\mathrm{p}>.10)$. There was no overall sex difference and no sex $\times$ litter size interaction (ps $>.10)$. Analysis of total play duration (lower panel, Figure 1) revealed a similar pattern of results. Play was strongly affected by 


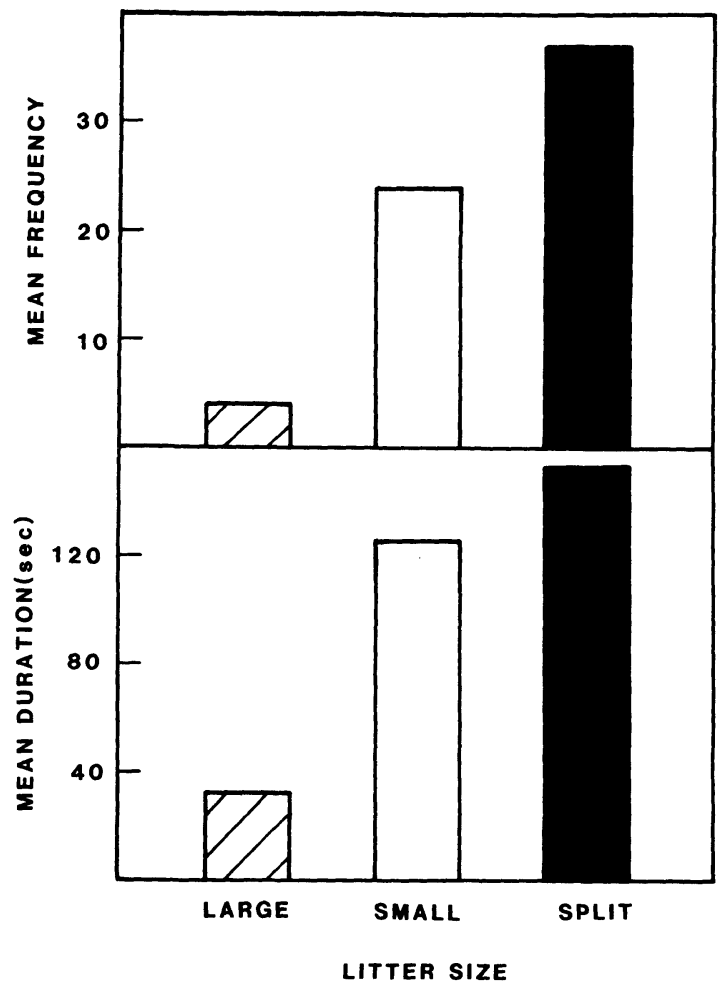

Figure 1. Mean total play frequency (upper panel) and duration (lower panel) in large $(N=8)$, small $(N=4)$, and split litters $(N=$ 4,4).

litter size $[\mathrm{F}(2,26)=9.21, \mathrm{p}<.005]$. Both small and split litters exhibited more play than the large litters (ps $<.05$ ). There was no significant difference, however, between small and split litters $(\mathrm{p}>.10)$. The frequencies of each individual play behavior summed across days were next examined by analyses of variance. Although these data were somewhat more variable, small litters exhibited significantly more of each play behavior $[\mathrm{Fs}(2,26)=3.95-17.26$, ps $<.05-.001]$, with the exception of a marginal increase in pouncing $[\mathrm{F}(2,26)=2.67$, $.05<\mathrm{p}<.10]$. Individual comparisons revealed that both small and split litters exhibited more of each play behavior than the large litter (ps $<.05)$, with the exception of pounce $(\mathrm{p}>.10)$. In only three behaviors (boxing, pinning, aggressive grooming) did the split litters significantly exceed the small litters ( $\mathrm{ps}<.05)$. The body weights of rats from small litters (mean $=122.6 \mathrm{~g}$ ) were significantly greater than those of the rats from the large litter [mean $=110.6 \mathrm{~g}, \mathrm{~F}(1,26)=5.86, \mathrm{p}<.025$ ] at the time of weaning. Although males (mean $=119 \mathrm{~g}$ ) weighed slightly more than females (mean $=113 \mathrm{~g}$ ), this difference was not statistically reliable $(p>.10)$.

\section{EXPERIMENT 2}

The results of Experiment 1 clearly show that play behavior is strikingly reduced in large litters housed in rela- tively small environments. The reversal of this effect in the split litter also indicates that play was strongly suppressed in the large litter. The most obvious explanation for these effects is that play is reduced by limited space. Since housing space was not manipulated in Experiment 1 , it seemed desirable to explore this variable directly. It also seemed of interest to compare the play of large and split litters reared in a larger environment to determine if more long-standing litter-size effects on play might emerge in a more spacious environment.

\section{Method}

Subjects. The subjects were four litters of rats derived from (Holtzman) female rats. The litters were reduced in size to eight (male $=4$, female $=4$ ) when the subjects were 10 days old. One litter was born and reared in a $50 \times 27 \times 31 \mathrm{~cm}$ aquarium (small), and a second litter in a $75 \times 32 \times 31 \mathrm{~cm}$ aquarium (medium). Two litters were born and reared in $100 \times 52 \times 48 \mathrm{~cm}$ environments (large) constructed of wood and wire screening. The subjects had ad-lib access to Purina Lab Chow and water. The subjects were weighed at 30 days of age, and the mothers were removed. One of the litters housed in the large environment (split litter) was divided into two groups of four (male $=2$, female $=2$ ) rats, and each group was housed in a large environment. Each rat was tailmarked for identification.

Apparatus and Procedure. The scoring, apparatus, and procedures were identical to those in Experiment 1.

\section{Results}

As in Experiment 1, initial analyses examined the frequency and duration of all scored play behaviors com-

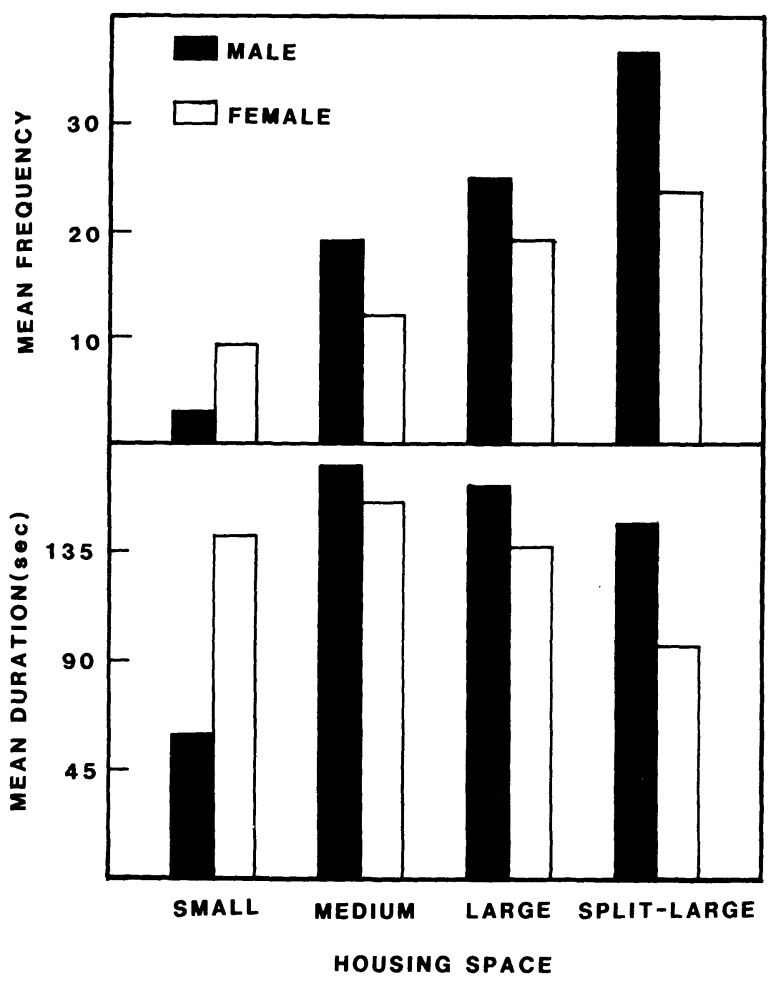

Figure 2. Mean total play frequency (upper panel) and duration (lower panel) of males and females in small, medium, large, and split-litter $(N=4,4)$ environments. 
bined. These data are summarized in Figure 2, which depicts the frequency (upper panel) and duration (lower panel) of play in male and female rats. It can be seen that play frequency increased in larger environments $[F(3,24)$ $=3.57, \mathrm{p}<.05]$. Individual comparisons revealed that the rats in the large environment played more than those in the small $(\mathrm{p}<.05)$, but there were no other significant group differences. There were no overall sex differences in play and no sex $\times$ environment size interaction (Fs < 1.0). There were no group or sex differences in the frequency of social grooming (Fs $<1.0$ ). There was, however, a marginally reliable interaction between sex and environment size $[\mathrm{F}(3,24)=2.86, .05<\mathrm{p}<.10]$ on grooming. This interaction reflects a reversal in sex differences as space increased from small (male, mean $=8.50$; female, mean $=13.0)$ to large $($ male, mean $=$ 11.2 ; female, mean $=7.5$ ) environments.

Play duration also increased as housing space increased $[F(3,24)=3.36, p<.05]$. Individual comparisons revealed that rats in the small environment played less than those in the medium and large environments (ps < .05). There was no difference between small- and split-litter rats, however $(\mathrm{p}>.10)$. Split-litter rats also played significantly less than those in the large environment $(\mathrm{p}$ $<.05)$. Analysis also revealed a significant sex $\times$ environment size interaction $[\mathrm{F}(3,24)=3.33, \mathrm{p}<.05]$. Males played substantially less than females in the small environment, but this sex difference was reversed in the split-litter rats (ps < .05). Comparison of the large- and split-litter groups (both housed in the large environment) reveals a slight decrease in play by the split-litter males $(9.4 \%)$ but a much more pronounced decline among females $(29.1 \%)$.

A series of analyses next examined housing-space effects on the frequency of each individual play behavior. Increased housing space increased the frequency of boxing, chasing, pinning, and aggressive grooming $[\mathrm{Fs}(3,24)$ $=3.03-6.84$, ps $<.01-.05]$. There were no significant differences in pouncing or wrestling $(\mathrm{ps}>.10)$. In none of these analyses were there sex differences or sex $\times$ environment size interactions ( $\mathrm{ps}>.10$ ).

At the time of weaning, males (mean $=117.1 \mathrm{~g}$ ) were heavier than females (mean $=100.4 \mathrm{~g}, \mathrm{p}<.002$ ), but there were no reliable differences among housing conditions $(\mathrm{p}>.10)$.

\section{DISCUSSION}

The fact that play increased when space was held constant and litter size was reduced (Experiment 1) or when litter size was held constant and space was increased (Experiment 2) clearly reveals that play in young rats is facilitated by increased housing space. Reversal of litter-size effects by the split litter of Experiment 1 also argues that restricted space produces a reversible suppression of play that (Experiment 2) affects males more strongly than females. Experiment 2 also suggests that, for litters of eight rats, increasing floor space to approximately $2,400 \mathrm{~cm}^{2}$ facilitates play but that further increases in space have only modest (males) or even suppressive (females) effects on this behavior. Clearly, then, levels of play in young male and female rats are strongly and differentially affected by amount of housing space available. One obvious interpretation of these space effects is a rather general suppression of behavior produced by restricted housing. In a series of additional observations (Klinger \& Kemble, 1984), however, we found that the groups tested in Experiment 1 did not differ in exploratory behavior, activity, or insect predation.

The fact that split-litter rats differed from rats in litters of comparable size (Experiment 1) and housing space (Experiment 2) suggests that litter size, presumably via differential social experience, may also have influenced the present results. Although the fact that this manipulation increased play in Experiment 1 but decreased it in Experiment 2 is puzzling, it is clear that these opposite effects could not represent a simple stress reaction to litter reduction or a simple release of play inhibition. It is, of course, possible that these differential effects could reflect an increase in available space to individual rats that approached (Experiment 1) and then exceeded (Experiment 2) optimal values for play. Thus, it is not possible to rule out a simple space-available hypothesis for the litter-size data. At the very least, however, the present data underscore the importance of these variables for future studies of play in rats.

\section{REFERENCES}

Davies, V. A., \& Kemble, E. D. (1983). Social play behaviors and insect predation in northern grasshopper mice (Onychomys leucogaster). Behavioral Processes, 8, 197-204.

Klinger, H. J., \& Kemble, E. D. (1984). Effects of litter size on exploratory behavior, insect predation, and activity. Unpublished manuscript.

KoepKe, J. E., \& Pribram, K. H. (1971). Effect of milk on the maintenance of sucking behavior in kittens from birth to six months. Journal of Comparative and Physiological Psychology, 75, 363-377.

Meany, M. J., \& STEWART, J. (1981). A descriptive study of social development in the rat (Rattus norvegicus). Animal Behaviour, 29, 34-45.

PanksePp, J., \& Beatty, W. W. (1980). Social deprivation and play in rats. Behavioral and Neural Biology, 30, 197-206.

THOR, D. H., \& Holloway, W. R. (1983). Play-solicitation behavior in juvenile male and female rats. Animal Learning \& Behavior, 11, 173-178.

(Manuscript received for publication August 27, 1984.) 